\title{
Transplante cardíaco na doença de Chagas
}

\author{
Heart transplantation in Chagas' disease
}

\author{
Layara Fernanda Lipari Dinardi', Jaqueline Fabiano Palazzo', Thiago Vicente \\ Pereira', Augusto Quaresma Coelho', Rebecca Rossener', Marcela Malheiro \\ Santos ${ }^{1}$, Vicente Pereira dos Santos Júnior², Bruno Canizares², \\ Alfredo Inácio Fiorelli ${ }^{3}$, Noedir Antônio Groppo Stolf ${ }^{4}$
}

Dinardi LFL, Palazzo JF, Pereira TV, Coelho AQ, Rossener R, Santos MM, Santos Júnior VP, Canizares B, Fiorelli Al, Stolf NAG. Transplante cardíaco na doença de Chagas / Heart transplantation in Chagas' disease. Rev Med (São Paulo). 2012;91(4):229-40.

\begin{abstract}
RESUMO: Dentre as diversas etiologias da insuficiência cardíaca, a miocardiopatia chagásica é considerada a mais agressiva. Como não há tratamento capaz de reverter a evolução da doença o transplante cardíaco torna-se a única opção. Foram analisados 107 pacientes portadores da doença de Chagas submetidos a transplante cardíaco, com idades

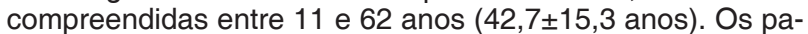
cientes portadores de megaesôfago e megacólon sintomáticos são automaticamente excluídos dos programas de transplante devido a uma maior possibilidade de complicações no pósoperatório a curto e longo prazo. A expectativa de resultados inferiores para o transplante em chagásicos em relação às demais cardiomiopatias não foi confirmada e, paradoxalmente, se encontram melhores taxas de sobrevida. Notou-se uma mortalidade imediata de $17,7 \%$ (19 casos), sendo as principais causas de morte: infecção (6 casos, $31,5 \%)$, disfunção do enxerto ( 6 casos, $31,5 \%$ ), rejeição ( 4 casos $21,1 \%$ ), parada cardiorrespiratória súbita (2 casos 10,5\%) e incompatibilidades ABO (1 caso 5,3\%). Tardiamente ao transplante, $27(25,2 \%)$ pacientes morreram, sendo as principais causas de morte: rejeição ( 6 casos, $22,2 \%$ ), infecção ( 6 casos, $22,2 \%$ ), linfoma( 4 casos, 14,8\%), Kaposi (2 casos, 7,4\%), pericardite constritiva ( 2 casos, $7,4 \%$ ) e reativação da doença de Chagas no sistema nervoso central (1 caso, 7,1\%). Por fim, pode-se concluir que: 1) o transplante cardíaco ainda é a única forma capaz de modificar a evolução natural da cardiomiopatia chagásica; 2) o diagnóstico precoce aliado à rápida introdução de benzonidazol leva a um reconhecimento de padrões histológicos normais do miocárdio sem que haja sequelas e 3) as doses de imunossupressores empregadas devem ser inferiores às utilizadas em outras etiologias.
\end{abstract}

DESCRITORES: Doença de Chagas; Cardiomiopatias; Transplante de coração; Rejeição de enxerto/prevenção \& controle; Imunossupressão; Transplante; Cardiomiopatia chagásica/diagnóstico.
ABSTRACT: Among the several etiologies of heart failure, the chagasic myocardiopathy is considered the most aggressive. Once there is no treatment capable of reverting the disease evolution, the heart transplantation becomes the only option. We analyzed 107 patients with Chagas disease submitted to heart transplantation, aged between 11 and 62 years (42.7 \pm 15.3 years). Patients with symptomatic megacolon and megaesophagus are automatically excluded from transplant programs due to a higher possibility of postoperative short and long term complications. The expectation of inferior results for the transplantation of chagasic patients in comparison with other myocardiopathies was not confirmed and, paradoxically, were found better survival rates. We noticed an immediate mortality rated in $17.7 \%$ (19 cases), whose main cause of death were: infection (6 cases, 31.5\%), graft dysfunction (6 cases, $31.5 \%$ ), rejection (4 cases $21,1 \%$ ), sudden cardiopulmonary arrest (2 cases $10.5 \%$ ) and ABO incompatibilities (1 case 5,3\%). Late after transplant, 27 (25.2\%) patients died, and the major causes were: rejection ( 6 cases, $22.2 \%$ ), infection (6 casos, $22.2 \%$ ), lymphoma (4 cases, $14.8 \%$ ), Kaposi sarcoma ( 2 cases, $7.4 \%$ ), constrictive pericarditis (2 cases, $7.4 \%$ ) and Chagas disease reactivation in the central nervous system (1 case, $7.1 \%)$. Finally, the conclusions are: 1) heart transplantation is still the only way to modify the natural course of chagasic myocardiopathy, 2) early diagnosis coupled to the rapid introduction of benzonidazol leads to a pattern recognition of normal myocardial histology without sequelae and 3) the doses of immunosuppressants used should be lower than those used in other etiologies.

KEYWORDS: Chagas disease; Cardiomyopathies; Heart transplantation; Graft rejection/prevention \& control; Immunosuppression; Transplantation; Chagas cardiomyopathy/ diagnosis.

1. Acadêmicos de Medicina, Faculdade de Medicina da Universidade de São Paulo.

2. Acadêmicos de Medicina, Faculdade de Medicina do ABC.

3. Diretor da Unidade de Perfusão Cardíaca e Assistência Cardiorrespiratória, Instituto do Coração do Hospital das Clínicas da Faculdade de Medicina da Universidade de São Paulo.

4. Professor, Instituto do Coração do Hospital das Clínicas da Faculdade de Medicina da Universidade de São Paulo.

Endereço para correspondência: Layara Fernanda Lipari Dinardi. Rua Arruda Alvim, ㄲo161 - apto 81 - Pinheiros, São Paulo SP. CEP: 05410-020. E-mail: layaralipari@gmail.com 


\section{INTRODUÇÃO}

A cardiopatia chagásica é considerada a pior etiologia dentre as diferentes formas de insuficiência cardíaca. Ela é a responsável pelo processo inflamatório crônico e persistente do miocárdio que leva à destruição de miocardiócitos, associado a arritmias e eventos embólicos, que são as principais causas de morte. A intensidade e a agressão da doença de Chagas diferem substancialmente daquela observada na miocardiopatia dilatada e, certamente, esses fatores são os principais responsáveis pela evolução que os pacientes apresentam em relação aos demais. Como não há tratamento algum comprovadamente eficaz para a cura ou capaz de conter a evolução da doença, o quadro progressivo é inevitável' ${ }^{1}$.

O único meio de reverter o quadro de pacientes com cardiomiopatia em fase terminal e restaurar suas condições hemodinâmicas é o transplante cardíaco (independentemente das considerações que possam ser feitas sobre o procedimento em um quadro infeccioso crônico). Depois do feito histórico realizado por Bernard em 1967, o transplante cardíaco passou a ser visto também como uma esperança para os portadores de cardiomiopatia chagásica ${ }^{2}$. Entretanto, foi necessário esperar 18 meses até que Zerbini, em um trabalho pioneiro, realizasse com êxito o transplante cardíaco em pacientes portadores de cardiomiopatia chagásica terminal com uma taxa de sobrevivência superior a 25 anos $^{3}$.

O transplante cardíaco na doença de Chagas difere fundamentalmente em relação às outras cardiomiopatias no que diz respeito aos imunossupressores, tendo em vista a possibilidade de reativação da doença e desenvolvimento de neoplasias. Se a princípio as indicações de transplante para chagásicos eram controversas, atualmente a escolha do imunossupressor e a estratégia de controle da reativação da doença mostram-se como as preocupações mais importantes.

\section{INDICAÇÃO E SELEÇÃO DOS PACIENTES}

A confirmação da etiologia chagásica como cardiomiopatia se apóia inicialmente em antecedentes epidemiológicos e na confirmação indireta da presença do parasita por meio de reações sorológicas para detecção de anticorpos anti-Trypanosoma cruzi, uma vez que a parasitemia é consideravelmente reduzida na fase crônica da doença. Na prática clínica, merecem destaque especial a reação de fixação de complemento (Reação de Machado-Guerreiro), considerada positiva quando a titulação é superior a 1:8, e a imunofluorescência indireta com titulação superior a 1:32. É incomum encontrar Trypanosoma cruzi no sangue periférico e, assim sendo, os testes que se utilizam dessa ferramenta pouco precisa raramente são empregados ${ }^{4,5}$.

Os critérios de indicação para o transplante na doença de Chagas pouco ou nada diferem dos adotados para outras cardiomiopatias. As principais causas de indicação são insuficiência cardíaca secundária em progressão da doença e mais raramente as arritmias incontroláveis. Em nossa casuística, dos 409 pacientes submetidos a transplante cardíaco, $107(26,2 \%)$ eram portadores da doença de Chagas, com idades compreendidas entre 11 e 62 anos (42,7 $\pm 15,3$ anos), e as principais indicações para o transplante foram insuficiência cardíaca (72 ou $67,2 \%$ ) em classe funcional IV (NYHA - New York Heart Association) e foram operados eletivamente. A fração de ejeção do ventrículo esquerdo determinada por ecografia bidimensional apresentava-se de $13 \%$ a $62 \%(32,4 \pm 9,8 \%)^{6}$.

A etiologia chagásica foi a terceira causa dentre as indicações para transplante, sendo precedida pelas cardiomiopatias dilatada e isquêmica, respectivamente. Os pacientes portadores de megaesôfago e megacólon sintomáticos são automaticamente excluídos dos programas de transplante devido a uma maior possibilidade de complicações no pósoperatório a curto e longo prazo.

Os pacientes operados em caráter de prioridade obedecem à seguinte distribuição: $33(30,8 \%)$ por choque cardiogênico, dos quais parte $(55,5 \%)$ estava em uso contínuo endovenoso de drogas vasoativas, outros $14(13,1 \%)$ em uso de balão intra-aórtico e $3(2,8 \%)$ estavam em assistência circulatória com ventrículo artificial. Finalmente, $3(2,8 \%)$ dos pacientes estavam em classe funcional II-III e a indicação principal do transplante foi a presença de arritmias malignas refratárias ao tratamento clínico.

Apesar da intensa evolução da doença, dificilmente os pacientes desenvolveram hipertensão pulmonar, o que talvez possa dar margem à explicação para o comprometimento biventricular da cardiomiopatia, apesar de esta não ser uma informação totalmente sustentada.

Achados de elevação da pressão na artéria pulmonar e da resistência vascular pulmonar estão muito mais ligados às péssimas condições hemodinâmicas dos pacientes e à congestão pulmonar. Tais alterações apresentam um caráter dinâmico, retrocedendo em poucos meses após o transplante com restauração do padrão hemodinâmico. Portanto, na cardiomiopatia chagásica a hiperreatividade pulmonar é esporadicamente o único critério de exclusão dos pacientes do programa de transplantes. Em nossa série, a pressão sistólica da artéria pulmonar variou 
entre 30 e $60 \mathrm{mmHg}(48,5 \pm 8,9 \mathrm{mmHg})$, o gradiente transpulmonar entre 9 e $32 \mathrm{mmHg}(11,6 \pm 7,9 \mathrm{mmHg})$ e a resistência vascular periférica entre 1,7 e 5,4 U Wood (3,6 $\pm 2,8 \cup$ Wood). Nota-se pela análise dos dados que há predomínio no padrão de congestão pulmonar em relação à hiperresistência ${ }^{7,8}$.

A disfunção ventricular direita muitas vezes se sobrepõe à esquerda no quadro clínico, havendo predomínio de congestão hepática, ascite e edema de membros inferiores. A congestão hepática congestiva crônica é associada a caquexia inerente à enfermidade e pode levar a disfunção hepática passiva crônica e renal com hipoalbuminemia e distúrbios de coagulação. Esta situação aumenta o risco cirúrgico do paciente à espera de transplante.

Em geral, os pacientes com doença de Chagas têm baixas taxas de hipersensibilização dos linfócitos, graças às transfusões de sangue ou cirurgias do coração necessárias graças à patologia em questão. O risco de hipersensibilização é maior em mulheres multíparas. A prova cruzada de linfócitos (Cross-match) é realizada rotineiramente, sendo analisada prospectivamente apenas nos pacientes que apresentam reatividade ao painel de linfócitos superior a $10 \%$. Mais recentemente, sabe-se que a tendência é usar a citometria de fluxo com uso de antígenos HLA purificados, o que permite a detecção e a caracterização dos anticorpos presentes no soro do receptor, sendo esta técnica conhecida como ucross-match virtuall. Esta característica tem uma grande importância de ordem prática, porque permite maior liberdade na busca à distancia do órgão para realização dos transplantes.

O componente arritmogênico na cardiomiopatia chagásica é muito exuberante devido à lesão do sistema de condução do coração, propiciando o desenvolvimento de bloqueios de ramo e do nó atrioventricular em graus variáveis. A presença de fibrilação atrial e extrassístoles ventriculares é frequente nas fases mais avançadas da doença. A presença de bloqueios em ramo direito e hemibloqueio anterior esquerdo não são patogênicos da doença, apresentam alta ocorrência na eletrocardiografia seriada realizada no seguimento da doença. A necessidade de estimulação cardíaca artificial na cardiomiopatia chagásica excede aquela observada para as demais cardiomiopatias.

$\mathrm{Na}$ nossa experiência, $34(31,8 \%)$ pacientes haviam recebido estimulação cardíaca artificial antes do transplante, dos quais $21(61,7 \%)$ eram portadores de marca-passo, $8(23,5 \%)$ de cardiodesfibrilador implantável, $3(8,8 \%)$ haviam recebido marca-passo e cardiodesfibrilador, sequencialmente, e um $(2,9 \%)$ havia recebido implante de células-tronco e terapia de ressincronização. O uso de cardiodesfibrilador implantável é extremamente útil nos portadores de arritmias complexas e sobreviventes de síncopes elétricas, tendo um papel de funcionamento também para ponte de transplante para os pacientes com tais características que estejam em lista de espera. A presença de parasitas e episódios de reativação da doença levam a uma miocardite transitória como o uso de benzonidazol. A necessidade de estimulação cardíaca artificial na doença de Chagas após o transplante, tanto imediata quanto tardiamente, não difere da observada em outras cardiomiopatias. Os fatores mais associados a indicação de estimulação cardíaca artificial pós-transplante estão muito mais relacionados a lesões sofridas pelo enxerto devido a anóxia e episódios de rejeição que à própria etiologia da cardiomiopatia ${ }^{7,8}$.

\section{TÉCNICA OPERATÓRIA}

O comprometimento biventricular observado na doença de Chagas gera maior propensão ao desenvolvimento de importantes cardiomegalias e, consequentemente, ao aumento da cavidade pericárdica. Como a colocação do coração do doador, com dimensões normais, não consegue ocupar totalmente a cavidade pericárdica e predispõe ao desenvolvimento de derrames pericárdicos de volumes variáveis, podendo requerir novas drenagens. A confecção de uma plicatura no saco pericárdico ou de uma janela pleuropericárdica podem apresentar benefícios relativos. A situação se torna mais crítica quando há também uma ascite volumosa que muitas vezes é espontaneamente drenada para o interior do saco pericárdico, retardando a retirada do dreno torácico.

Nos casos mais graves, com disfunção ventricular direita severa, a presença de ascite muito volumosa nas cúpulas diafragmáticas, que podem estar excessivamente elevadas, dificultando o implante do enxerto, devendo muitas vezes ser realizado um esvaziamento parcial para facilitar a realização das anastomoses. A drenagem total ou excessiva está contra-indicada por aumentar a possibilidade de ocorrência de distúrbios hidroeletrolíticos imediatamente no pós-operatório. Com a melhora hemodinâmica, a ascite tende a se extinguir espontaneamente poucos meses após o transplante.

É necessário ter um cuidado especial em relação à veia cava superior pela presença de múltiplos cabos de eletrodos que muitas vezes podem provocar firme aderência, aumentando a predisposição às lesões venosas ou à retração da anastomose, podendo levar a variados graus de estenose. Outro cuidado necessário é prestar uma atenção especial à manipulação do coração antes do pinçamento da aorta, 
graças à maior possibilidade de desprendimento de trombos para o território pulmonar ou sistêmico.

Em relação à técnica operatória propriamente dita, não há grandes divergências em relação ao transplante nas demais cardiomiopatias. No inicio de nosso experimento, foi utilizada a técnica clássica proposta por Lower e Shamway ${ }^{9}$, com anastomose biatrial. Posteriormente, com a introdução da técnica bicaval, seguindo a orientação de muitos, o transplante passou a ser realizado segundo essa nova modalidade, mantendo a anastomose uniatrial esquerda ${ }^{9,10}$. Em 2002, foi introduzida a anuloplastia profilática de tricúspide com a finalidade de minimizar os efeitos tardios da regurgitação valvar, sendo que os resultados iniciais do experimento foram previamente publicados. O método foi utilizado em 32 (29,9\%) pacientes da série anteriormente citada ${ }^{11}$.

\section{CORAÇÃO NATIVO}

O parasita é encontrado no miocárdio mais frequentemente na fase aguda da doença ou duran- te os episódios de reativação. Na fase crônica há predomínio do aumento de tamanho das câmaras cardíacas, a identificação do parasita nem sempre ocorre e a reação inflamatória é menos intensa e há substituição de miocárdio por fibrose. É freqüente a presença de uma lesão na ponta do ventrículo esquerdo com adelgaçamento da parede por atrofia muscular sendo que em alguns casos pode levar à formação de um aneurisma apical. Este tipo de lesão não é patognomônica da doença de Chagas, mas é um achado muito característico.

Em nossa casuística, dos 103 corações explantados durante os transplantes, o exame anátomopatológico evidenciou miocardite em 103 (96,3\%) dos casos, presença de afinamento da ponta do ventrículo em $70(65,4 \%)$, trombos intracavitários em $36(33,6 \%)$ e detecção de formas amastigotas do parasita Tripanosoma cruzi em 21 (19,6\%). Na Figura 1 se encontram representados os principais achados anátomo-patológico dos corações extraídos durante o transplante.

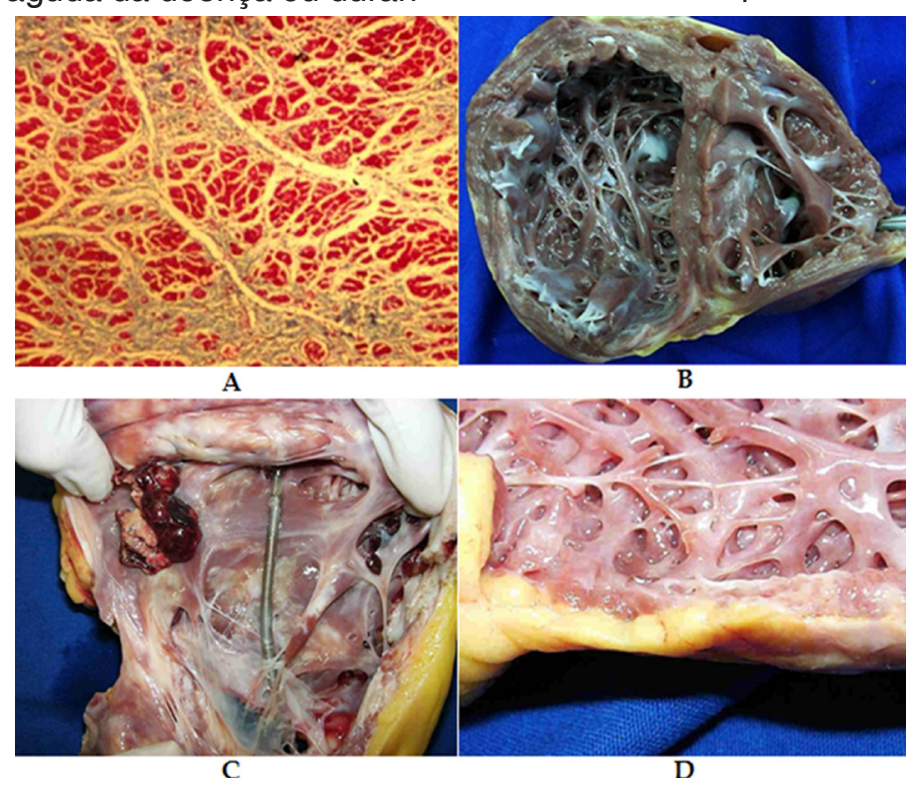

Figura 1. A) Presença de miocardite crônica com atividade inflamatória variável e intenso processo de fibrose em substituição ao miocárdio normal; B) Corte transversal do coração, evidenciando as cavidades ventriculares; C) Vista inferior do ventrículo direito com a presença de eletrodos do marca-passo, extensas áreas de fibrose difusa em grandes trombos murais; D) Vista da parede do ventrículo esquerdo exibindo a lesão apical extensa, onde se observa intensa atrofia muscular

O exame anátomo-patológico dos corações dos pacientes que vieram a óbito ao final do transplante não exibe as alterações morfológicas observadas na primeira fase crônica da doença, apesar das reativações ocorridas (Figura 1). Tais informações sugerem que a presença de imunossupressão massiva própria do transplante deve alterar o comportamento natural da inflamação crônica sobre o miocárdio, o que o torna mais resistente a processos necróticos e desarranjo de fibrose. Sabe-se que a imunossupressão aumenta a predisposição a parasitemia e, por outro lado, aumenta a tolerância do paciente ao benzonidazol, mas sua interferência na inflamação crônica sobre o miocárdio, a longo prazo, necessita de mais estudos.

\section{IMUNOSSUPRESSÃO}

O transplante cardíaco na doença de Chagas 
sempre representou um grande desafio devido à possibilidade de reativação da doença e de manutenção da imunossupressão sobre uma doença infecciosa crônica. Os conhecimentos acumulados ao longo de mais de 25 anos permitem identificar basicamente três períodos distintos.

O protocolo inicial de imunossupressão não difere substancialmente daquele aplicado aos pacientes portadores de outras cardiomiopatias, consistindo no esquema triplo de ciclosporina, corticóide e azatioprina. A estratégia quanto ao uso de benzonidazol foi igualmente discutida e aprovada. O uso profilático do fármaco no pré-operatório teve como principal obstáculo definir qual era o momento exato do seu início, uma vez que o transplante não ocorre de forma eletiva. O uso profilático no pós-operatório imediato encontrou maior sustentabilidade pela maior facilidade de controle e coincidia com o início da imunossupressão. Surpreendentemente ambos os grupos apresentaram reativação da doença e recorreu-se à pulsoterapia para o controle da rejeição celular mais exacerbada. Além disso, descobriu-se que a resolução dos episódios de reativação da doença não impede o aparecimento de outros, estando muito mais relacionado com o processo de imunossupressão do paciente.

Chamava a atenção que a miocardite própria da reativação da doença, uma vez instalada, não deixou sequelas funcionais no miocárdio e foi observado o desaparecimento do intenso processo inflamatório em duas ou três semanas através da biópsia endomiocárdica de rotina. $\mathrm{O}$ evento inesperado foi o início precoce de neoplasias, como o linfoma, que estão vinculadas com o uso da ciclosporina devido à sua ação de bloqueio nos linfócitos $T$.

No segundo período já estava claro que a indicação profilática de benzonidazol não tinha mais suporte para o uso, passando a ser indicado somente nos períodos de recaída. O nifurtimox na dose de 8 a
$10 \mathrm{mg} / \mathrm{kg} / \mathrm{dia}$ e o alopurinol na dose de 600 a $900 \mathrm{mg}$ foram igualmente provados por outros grupos e podem ser utilizados com eficiência similar.

A terceira fase coincide com a consolidação do transplante cardíaco como padrão-ouro no resgate de pacientes com cardiomiopatia terminal. Foi proposta a substituição da azatioprina pelo micofenolato de mofetil, respeitando-se o mesmo protocolo utilizado para os demais pacientes. No entanto, os resultados foram decepcionantes, porque pacientes que se encontravam estáveis em fase tardia do transplante voltavam a apresentar reativação da doença. Provavelmente com o uso de metade da dose utilizada de fármaco seja possível obter os benefícios da imunossupressão associada a sua ação anti-proliferativa.

$\mathrm{Na}$ nossa experiência os $10(9,3 \%)$ primeiros pacientes receberam $1 \mathrm{~g}$ endovenoso de metilprednisona até a introdução da dieta oral e, posteriormente, $1 \mathrm{mg} / \mathrm{kg} / \mathrm{dia}$ com redução gradual até $0,2 \mathrm{mg} / \mathrm{kg} / \mathrm{dia}$. A azatioprina foi inicialmente administrada de 1,5 a $3,5 \mathrm{mg} / \mathrm{kg} / \mathrm{dia}$ e ajustada em função da contagem de leucócitos ou da incidência de rejeição, exceto em $1(0,9 \%)$ paciente que não recebeu azatioprina. A ciclosporina foi administrada por via endovenosa durante a operação na dose de 1 a $2 \mathrm{mg} / \mathrm{kg} / \mathrm{dia}$, buscando-se manter valores no sangue entre $700 \mathrm{a}$ $1200 \mathrm{ng} / \mathrm{ml}$ e reduzindo para 500 e $700 \mathrm{ng} / \mathrm{ml}$ depois do primeiro trimestre.

Posteriormente, a partir de 1992, as doses iniciais de corticóide foram mantidas nas mesmas proporções, mas com redução mais acentuada e precoce em função da evolução do paciente. A dose de azatioprina foi elevada para $3 \mathrm{mg} / \mathrm{kg} / \mathrm{dia}$ e houve redução da ciclosporina com manutenção dos valores plasmáticos entre 200 e $300 \mathrm{ng} / \mathrm{ml}$ na fase imediata ao transplante e entre 100 e $150 \mathrm{ng} / \mathrm{ml}$ na fase tardia ${ }^{7,8}$ (Figura 2).

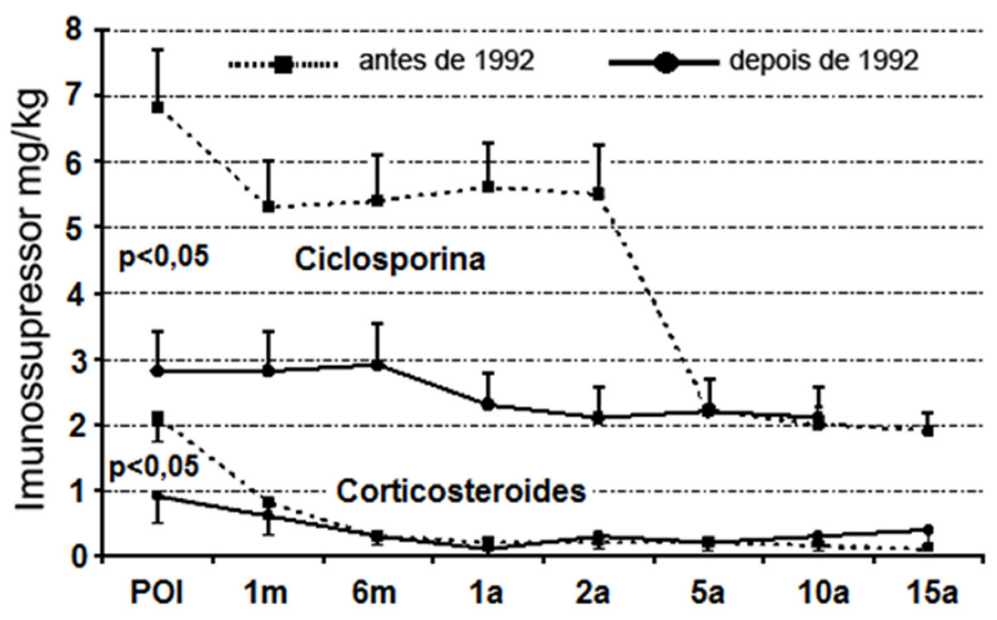

Figura 2. Variação na dosagem de ciclosporina e corticóide em função da primeira e da segunda fase. Nota-se redução significativa em ambos imunossupressores. POI: pós-operatório imediato, m: meses e a: anos 
A partir de 2001 um grupo de 15 (14\%) pacientes recebeu $2 \mathrm{~g}$ de micofenolato de mofetil (MMF) ao invés de azatioprina (Tabela 1). Apenas 1,8\% dos pacientes receberam tacrolimus por intolerância à ciclosporina. A globulina antitimocítica foi administrada por via endovenosa em $13(12,1 \%)$ pacientes na dose de 10 a 15mg/ $\mathrm{kg} / \mathrm{dia}$ durante cinco a dez dias ${ }^{12}$. Observações semeIhantes foram feitas por outros autores brasileiros ${ }^{13}$.

Tabela 1. Distribuição das doses iniciais dos imunossupressores utilizados no transplante cardíaco em portadores da doença de Chagas nas diferentes épocas

\begin{tabular}{l|cccc}
\hline \multicolumn{1}{c|}{ Eras } & 1985 a 1992 & 1992 a 2001 & 2001 & 2001 a 2010 \\
\hline Número de Pacientes & 10 & 38 & 15 & 44 \\
\hline Ciclosporina (mg/Kg/dia) & $6,8 \pm 2,0$ & $2,8 \pm 1,0$ & $2,9 \pm 1,4$ & $2,9 \pm 1,3$ \\
\hline Azatioprina (mg/Kg/dia) & $1,4 \pm 1,3$ & $2,7 \pm 0,8$ & - & $2,9 \pm 0,9$ \\
\hline MMF (g/dia) & - & - & $2,0 \pm 0,5$ & - \\
\hline Corticóide (mg/Kg/dia) & $2,1 \pm 1,3$ & $0,9 \pm 0,8$ & $0,8 \pm 0,9$ & $0,8 \pm 0,8$ \\
\hline$P$ & $<0,05$ & & $>0,05$ & \\
\hline
\end{tabular}

MMF - Micofenolato de Mofetil

\section{REATIVAÇÃO DA DOENÇA}

A reativação da infecção por Trypanosoma cruzi é um problema real que só encontrará solução com a descoberta de uma maneira segura de eliminar o parasita do paciente. Por outro lado, o transplante cardíaco na doença de Chagas tornou-se uma realidade a ser praticada, sendo que os pacientes requerem cuidados especiais no período pós-operatório.

A reativação na doença de chagas é igual a que ocorre em qualquer infecção crônica, podendo se manifestar em qualquer estado clínico ou farmacológico que leve o hospedeiro a um estado de imunossupressão acentuada. Essa reativação tem sido muito descrita em pacientes portadores da síndrome da imunodeficiência adquirida, neoplasias com tratamento quimioterápico e outras enfermidades imunossupressoras ${ }^{14-17}$. No caso do transplante cardíaco, a reativação do Trypanosoma cruzi pode ocorre em qualquer tecido do organismo, sendo mais freqüente no coração, subcutâneo, sangue, fígado, cérebro e outros ${ }^{18,19}$.

O uso de órgãos nos portadores da doença de Chagas é completamente contra-indicado por total falta de controle da doença nos receptores e, portanto, nos sensibilizados. Em nossa experiência tivemos um paciente portado de cardiomiopatía idiopática que recebeu inadvertidamente um coração supostamente normal, porém o doador era portador de Trypanosoma cruzi sem manifestações clínicas de tal doença. O paciente recebeu benzonidazol de maneira profilática nos pós-operatório imediato e foram acompanhados com a maior atenção em virtude da possibilidade de reativação da doença, sem que houvesse apresentado qualquer alteração laboratorial ou achados anormais em biópsias. O uso de órgãos de doadores chagásicos é realizado em situações muito especiais ${ }^{20,21}$.

Por outro lado, Kun et al., descobriu a presença da reativação da doença de Chagas em pacientes que também receberam corações supostamente normais, sem embargo, sendo que depois a operação mostro uma disfunção grave do enxerto com miocardite aguda seguida de morte. O estudo anatomopatológico confirmou a presença do Trypanosoma cruzi no miocárdio ${ }^{22}$. Essas observações confirmaram que a resposta ao parasita no hospedeiro não é uniforme, sendo que os seguintes fatores apresentam uma dada importância, como a forma do parasita, o estado imunológico do paciente, as condições da cirurgia, a estratégia de tratamento, entre outros.

Nos exames laboratoriais a identificação indireta do parasita isolado, tais como reações de fixação de complemento e determinação de antígeno protéico IgG se mostra totalmente ineficaz no diagnóstico da reativação da doença por Trypanosoma cruzi, bem como um fator preditivo de diagnóstico precoce. $\mathrm{Na}$ nossa experiência o diagnóstico de reativação 
da doença foi realizado através da identificação do parasita no miocárdio, nos nódulos cutâneos, no sangue ou por testes sorológicos. O xenodiagnóstico e o cultivo do sangue não foram considerados para o diagnóstico de reativação da doença em virtude das possibilidades de falsos positivos. Os episódios de parasitemia ou reativação da doença de Chagas foram tratados com benzonidazol em duas doses de $10 \mathrm{mg} / \mathrm{Kg} /$ dia durante 60 dias.

Em geral cerca de $20 \%$ a $30 \%$ dos pacientes costumam apresentar ao menos um episódio de reativação da doença de Chagas ao longo da vida.
Apesar de a reativação poder ocorrer a qualquer momento, a maior incidência foi verificada no primeiro ano depois do transplante, quando a imunossupressão está mais acentuada em virtude da ocorrência de rejeição celular.

A Figura 3 apresenta a curva livre de reativação da doença de Chagas, comparando os pacientes da primeira e segunda fase da experiência, ou seja, com as doses convencionais de imunossupressão e com doses reduzidas. A análise das curvas demonstrou uma redução significativa da reativação acompanhando a diminuição da imunossupressão.

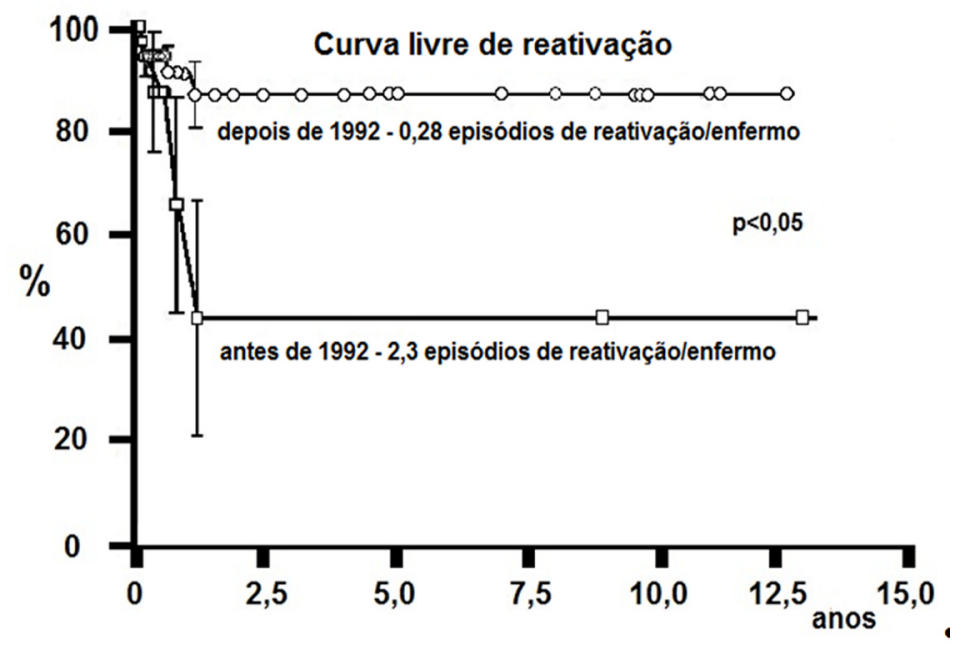

Figura 3. Curva livre de reativação da doença de Chagas depois do transplante, nota-se redução significativa na $2^{a}$ Fase do experimento clínico

Vinte (19,6\%) pacientes apresentaram reativação da doença de Chagas, totalizando 32 episódios, com variação de um a oito episódios por paciente. $\mathrm{Na}$ primeira fase da experiência a medida de reativação foi de 2,3 episódios por paciente, sendo reduzida na segunda fase a 0,28 episódios por paciente. $\mathrm{O}$ diag- nóstico de reativação da doença de Chagas foi determinado pela biópsia endomiocárdica em $23(71,8 \%)$ episódios, por xenodiagnóstico em 11 (34,3\%), pela biópsia de nódulos subcutâneos (25\%), por biópsia do sistema nervoso central (Figura 4$)$ em $1(3,1 \%)$ e fígado em 1 (3,1\%).

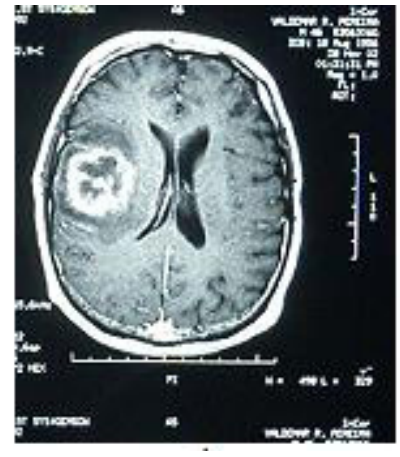

A

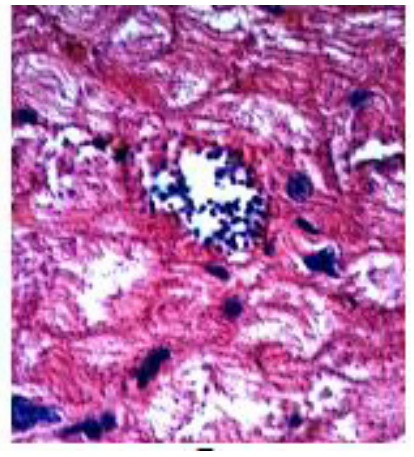

$\overline{\mathrm{B}}$

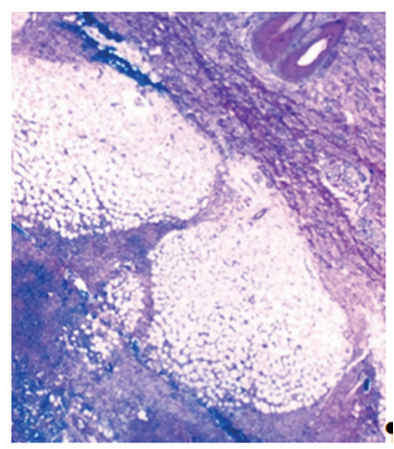

C

Figura 4. A) Ressonância magnética do crânio de um paciente que apresentou imagem nodular em região parietal direita, expansiva com compressão dos tecidos circundantes e obliteração dos sulcos. A biópsia do tecido confirmou a presença do parasita no sistema nervoso central; B) Presença de formas amastigotas no miocárdio identificado em biópsia de rotina; C) Presença de reação inflamatória no tecido celular subcutâneo, paniculite, em função da presença do parasita 
As manifestações clínicas da reativação são inespecíficas, podendo-se observar: fibrose em $16,6 \%$, arritmias em $11,1 \%$, insuficiência cardíaca em $5,5 \%$ e hepatomegalia em 5,5\%. A Figura 4 apresenta as diferentes formas de identificação dos parasitas nos tecidos durante a reativação da doença.

\section{REJEIÇÃO}

O controle da rejeição celular foi realizado inicialmente por biópsia endomiocárdica e posteriormente com auxilio da gammagrafía cardíaca com Gálio, conforme o protocolo previamente publicado ${ }^{23}$.
A pulsoterapia com corticóides está indicada em casos de rejeição com grau superior a 2, de acordo com a classificação da International Society for Heart and Lung Transplantation ${ }^{24}$.

A distribuição da severidade dos episódios de reativação da doença de Chagas segue as mesmas proporções observadas nos transplantes das demais cardiopatias. No prezado momento, as rejeições que requerem pulsoterapia são consideradas fatores de risco para reativação por acentuarem a imunossupressão. A Figura 5 demonstra a íntima relação entre o número de rejeição e os episódios de reativação da infecção por Trypanosoma cruzi.
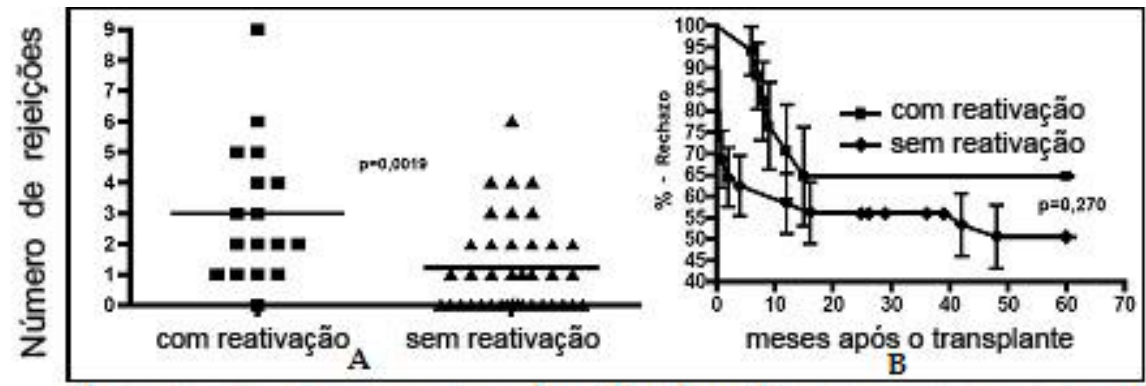

Figura 5. Pode-se observar a relação direta estabelecida entre o núméro de rejeições e os episódios de reativação da infecção por Trypanosoma cruzi

No caso específico da Doença de Chagas muitas vezes é difícil diferenciar o processo inflamatório secundário à miocardite produzida pela reativação do Trypanossoma cruzi daquela secundária à rejeição celular aguda. Os processos inflamatórios podem ser confundidos e as amostras de miocárdio serem pequenas, sendo que nem sempre é possível identificar o parasita. Dessa forma a detecção do parasita no miocárdio é essencial para a confirmação do diagnóstico de reativação da doença. Mais recentemente foi utilizada a técnica de PCR para o Trypanossoma cruzi em amostras de miocárdio com o objetivo de auxiliar no diagnóstico diferencial e como fator preditivo da reativação. Outros métodos se encontram em avaliação e espera-se que em um futuro próximo obtenha-se resultados mais consistentes que possam colocar o exame em um lugar real dentro do arsenal de diagnósticos ${ }^{25,26}$. Os corticóides são considerados um fator de risco importante para a reativação da Doença de Chagas, a tal ponto que muitos autores preferem não usá-los na imunossupressão, obtendo bons resultados ${ }^{27}$. O alopurinol foi proposto como um método alternativo para controlar a reativação da doença ${ }^{28}$.

\section{NEOPLASIAS}

A probabilidade do desenvolvimento das neoplasias nos pacientes submetidos a transplante de órgãos sólidos é cerca de quatro vezes maior em relação à população em geral, o que torna a imunossupressão um fator de risco. Na era pré-ciclosporina, os pulmões e a pele predominavam e, posteriormente, deram lugar aos linfomas, muito provavelmente por um intenso bloqueio dos linfócitos T promovido por ação da ciclosporina sobre a interleucina-2.

Acredita-se que este bloqueio interfira nos elementos que controlam a proliferação de linfócitos $B$ e que é potencializado em diferentes fatores, tais como: infecções virais, reativação de vírus latentes, episódios de reativação e pelo próprio benzonidazol, que gera predisposição para o aparecimento de linfomas. Sabe-se que o benzonidazol é capaz de alterar a resposta imunológica, provavelmente por seu efeito citotóxico sobre os linfócitos $T$, que pode atuar de maneira sinérgica com a ciclosporina e a infecção por Trypanosoma cruzi, potencializando a agressividade e a precocidade no aparecimento de neoplasias.

As características dos pacientes do nosso estudo com doença de Chagas submetidos a transplante cardíaco que desenvolveram neoplasias estão representadas na Tabela 2. Deve-se notar que a doença linfoproliferativa e o sarcoma de Kaposi formam as neoplasias mais frequentes e se comportam de formas extremamente agressivas, porque se desenvolvem precocemente e todos os pacientes afetados morreram. O tempo médio de aparecimento desta forma de neoplasia foi apenas 6,5 $\pm 2,1$ meses depois do 
transplante. O número de reativações da doença de Chagas, antes de se observar neoplasia de Kaposi e linfomas, variou em 1 a 4 episódios com média de $2,3 \pm 0,89$ por paciente.

Tabela 2. Características dos pacientes com doença de Chagas e submetidos a transplante cardíaco que desenvolveram neoplasias

\begin{tabular}{l|c|c|l}
\hline \multicolumn{1}{c|}{ Tipo de Neoplasia } & $\begin{array}{c}\text { Aparecimento da neoplasia } \\
\text { (meses) }\end{array}$ & $\begin{array}{c}\text { Número de reativações antes da } \\
\text { neoplasia (meses) }\end{array}$ & Evolução \\
\hline Kaposi $^{*}$ & 96 & 2 & Morte \\
\hline Linfoma* $^{*}$ & 4 & 2 & Morte \\
\hline Linfoma $^{*}$ & 6 & 4 & Morte \\
\hline Carcinoma Espinocelular $^{*}$ & 60 & 0 & Vivo \\
\hline Linfoma $^{*}$ & 9 & 2 & Morte \\
\hline Linfoma $^{*}$ & 7 & 3 & Morte \\
\hline Kaposi & & 1 & Morte \\
\hline
\end{tabular}

Legenda: * - 1a Fase $e^{* *}-2 a$ Fase.

Na Figura 6 está representado o tumor de Kaposi, identificado inicialmente nos pulmões e em um caso de linfoma intestinal. Na Figura 7 está representada a curva livre de neoplasias compa- rando dois períodos distintos com protocolos de imunossupressão diferentes, notando-se uma significativa redução das neoplasias com a diminuição da imunossupressão.

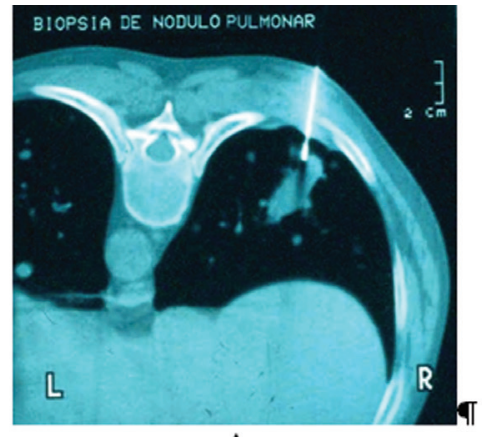

$\mathrm{Ao}$

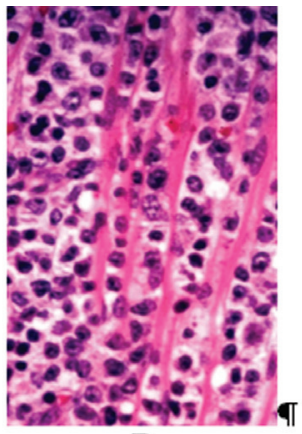

$\mathrm{B}$.

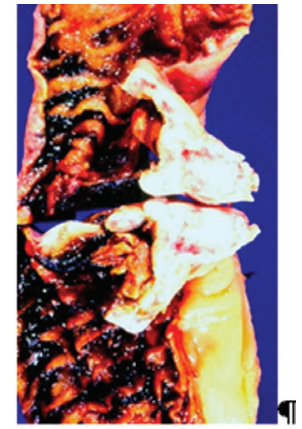

$\mathrm{Co}$

Figura 6. A) Paciente com nódulo pulmonar cuja biópsia percutânea revelou a presença de um tumor de Kaposi; B) Aspecto histológico do tumor de Kaposi; C) Paciente no 6ํㅡês pós-transplante que desenvolveu enterorragia e sinais de abdome agudo. Na operação foi evidenciada a presença do tumor na parede do intestino delgado, sendo realizada enterectomia e anastomose. A ciclosporina foi suspensa e 3 meses depois o paciente faleceu devido a uma infecção. A necropsia não mostrou recidiva do tumor

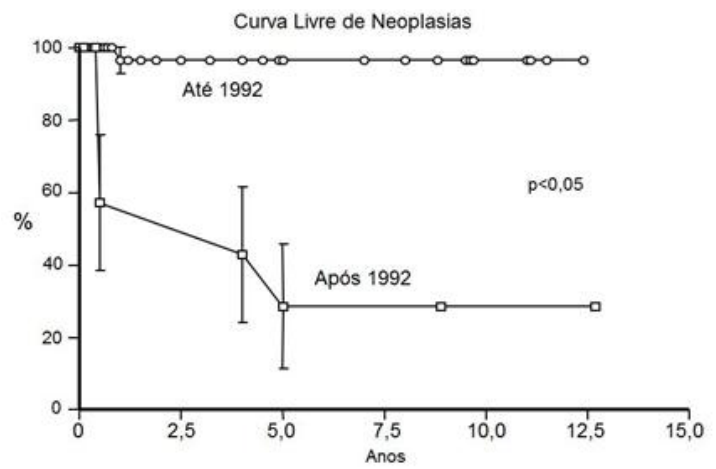

Figura 7. Curva livre de neoplasia depois do transplante (nota-se que há redução) 


\section{INFECÇÃO}

Os processos infecciosos não diferem significativamente dos observados nos demais pacientes submetidos a transplante cardíaco, havendo predomínio de infecções virais (40\%-45\%), bacterianas (35\%-40\%) e pelo protozoário Pneumocistis carini (10\%-15\%), sendo que esta distribuição apresenta pouca variação nas diferentes instituições.

A ocorrência de neoplasias nesta série está estreitamente relacionada com infecção por citomegalovírus, possivelmente porque os pacientes estudados apresentaram previamente alta incidência de IgG positivo para o vírus, ao contrário dos que se observaram na população geral.

\section{RESULTADOS}

Estudos multicêntricos com transplante cardíaco em portadores de cardiomiopatia chagásica têm mostrado, de maneira paradoxal, que, em comparação com as outras etiologias, a sobrevida destes pacientes tende a melhorar após a cirurgia, apesar de a enfermidade representar um fator de risco para os pacientes em lista de espera ${ }^{29-31}$. É possível que a baixa reatividade vascular pulmonar própria desta doença e a maior atenção dedicada no pós-operatório em função da possibilidade de reativação possam desempenhar um papel favorável no acompanhamento destes pacientes. Depois do transplante, a melhoria da qualidade de vida pode ser igualmente observada nos diferentes centros.

A indicação do transplante na cardiomiopatia chagásica foi inicialmente muito discutida e controversa, porque a doença, que apresenta caráter sistêmico, tem origem infecciosa e não é possível efetuar uma esterilização do parasita.
No entanto, rapidamente os resultados iniciais confirmaram suas vantagens e o método passou a representar a alternativa mais eficiente para resgatar os pacientes em estágio final da cardiomiopatia. A expectativa de resultados inferiores para o transplante em chagásicos em relação às demais cardiomiopatias não foi confirmada e, paradoxalmente, se encontram melhores taxas de sobrevida, provavelmente pela resistência pulmonar mantida e por se tratar de um grupo à parte que requeria uma atenção especial (devido ao risco de reativação).

Em nossas observações, notou-se uma mortalidade imediata de $17,7 \%$ (19 casos), sendo as principais causas de morte: infecção (6 casos, 31,5\%), disfunção do enxerto ( 6 casos, $31,5 \%$ ), rejeição (4 casos $21,1 \%$ ), parada cardiorrespiratória súbita (2 casos $10,5 \%$ ) e incompatibilidades ABO (1 caso $5,3 \%$ ). Dos três pacientes que estavam em uso de assistência circulatória mecânica, dois estavam vivos e estáveis, e o terceiro faleceu no pós-operatório imediato por hemorragia.

Tardiamente ao transplante, $27(25,2 \%)$ pacientes morreram, sendo as principais causas de morte: rejeição (6 casos, 22,2\%), infecção (6 casos, $22,2 \%$ ), linfoma( 4 casos, $14,8 \%$ ), Kaposi (2 casos, $7,4 \%$ ), pericardite constritiva ( 2 casos, $7,4 \%$ ) e reativação da doença de Chagas no sistema nervoso central (1 caso, $7,1 \%)$.

Quando os pacientes foram divididos em dois grupos, antes e depois de 1992, em função da troca da imunossupressão, notou-se que na segunda fase houve melhora significativa de sobrevida, como se observa na Figura 8. Comparando-se as curvas de sobrevida dos pacientes com cardiomiopatia chagásica em relação ao grupo geral, pode-se observar que há uma tendência não significativa de melhores resultados no primeiro grupo, conforme apresentado na Figura 9.

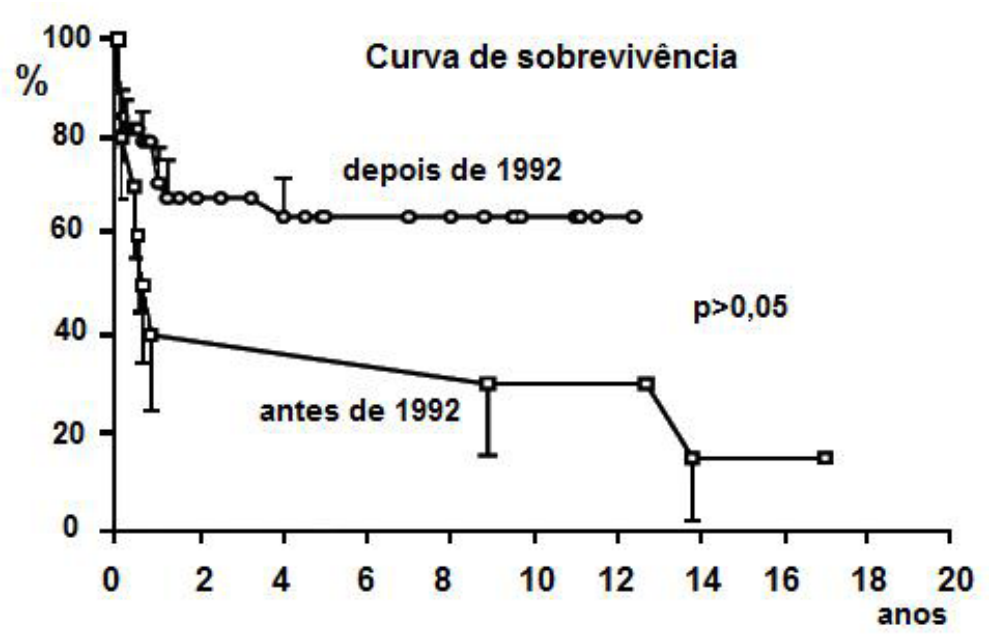

Figura 8. Curvas de sobrevida comparando a $1^{\text {a }}$ e a $2^{a}$ fase. Nota-se melhora significativa com redução dos imunossupressores 


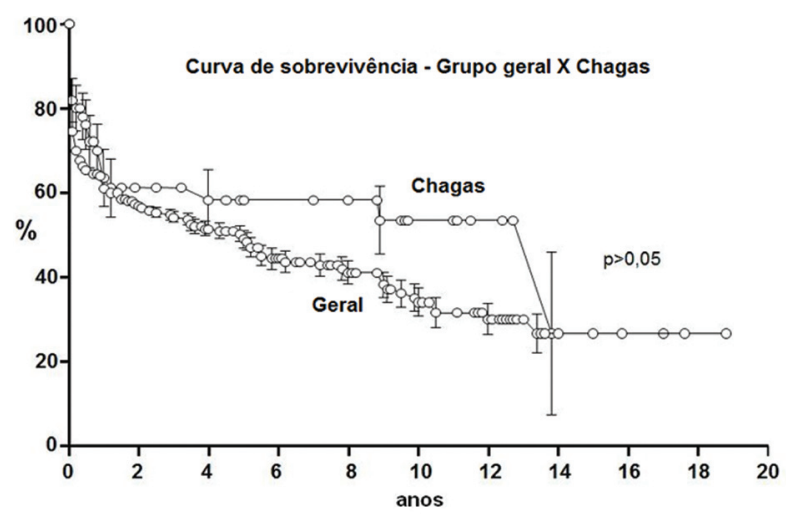

Figura 9. Curva de sobrevida dos pacientes submetidos a transplante cardíaco, comparando etiologia chagásica e geral, observando-se a tendência não significativa de melhores resultados no primeiro grupo

\section{CONCLUSÕES}

Atualmente, o transplante cardíaco para a doença de Chagas é uma realidade. Ainda que os portadores de tal enfermidade apresentem várias complicações com outras etiologias, na prática, estas dificuldades são bem conhecidas e controladas com estratégias de prevenção e bem patrocinadas.

O transplante cardíaco na cardiomiopatia chagásica está revestido de desafios peculiares que divergem em relação às demais etiologias pela possibilidade de reativação da doença e surgimento de neoplasias. Contudo, o transplante cardíaco é a única forma que existe atualmente capaz de modificar

\section{REFERÊNCIAS}

1. Rassi Jr A, Rassi1 A, Marin-Neto JA. Chagas heart disease: pathophysiologic mechanisms, prognostic factors and risk stratification. Mem Inst Oswaldo Cruz, Rio de Janeiro. 2009;104(supl. I):152-8.

2. Barnard CN. The operation. A human cardiac transplant: an interim report of a successful operation performed at Groote Schuur Hospital, Cape Town. S Afr Med J. 1967;41(48):1271-4.

3. Zerbini EJ, Décourt LV. Experience on three cases of human heart transplantation. Laval Med. 1970;41(2):14954.

4. Rassi A Jr, Rassi A, Marin-Neto JA. Chagas disease. Lancet. 2010;375(9723):1388-402.

5. Coura JR, Borges-Pereira J. Chagas disease: 100 years after its discovery. A systemic review. Acta Trop. 2010;115(1-2):5-13.

6. Freitas HF, Nastari L, Mansur AJ, Bocchi EA, Moreira a evolução natural da doença e resgatar o paciente que está em fase terminal. O método é considerado também como padrão para restaurar as condições hemodinâmicas do paciente com insuficiência cardíaca secundária ao processo evolutivo da doença.

O diagnóstico precoce e a rápida introdução de benzonidazol são capazes de reconhecer os padrões histológicos normais do miocárdio sem deixar sequelas. A imunossupressão, na reativação da doença, exige, portanto, uma atenção especial quanto ao uso de fármacos. Os cuidados mais importantes a se tomar são com as doses de imunossupressores, que devem ser diferentes e inferiores às utilizadas em outras etiologias.
LF, Bacal F, Barretto AC, Stolf NA, Bellotti G. Dynamics of patient selection for heart transplantation or cardiomyoplasty. Arq Bras Cardiol. 1994;62(4):233-7.

7. Fiorelli AI, Stolf NA, Honorato R, Bocchi E, Bacal F, Uip D, Strabelli T, Issa V, Amato VA, Fiorelli LR, Oliveira SA. Later evolution after cardiac transplantation in Chagas' disease. Transplant Proc. 2005;37(6):2793-8.

8. Fiorelli Al, Santos RH, Oliveira JL Jr, Lourenço-Filho DD, Dias RR, Oliveira AS, da Silva MF, Ayoub FL, Bacal F, Souza GE, Bocchi EA, Stolf NA. Heart transplantation in 107 cases of Chagas' disease. Transplant Proc. 2011;43(1):220-4.

9. Lower RR, Shumway NE. Studies on orthotopic transplantation of the canine heart. Surg Forum. 1960;11:18.

10. Dreyfus G, Jebara V, Mihaileanu S, Carpentier AF. Total orthotopic heart transplantation: an alternative 
to the standard technique. Ann Thorac Surg. 1991;52(5):1181-4.

11. Fiorelli Al, Stolf NA, Abreu Filho CA, Santos RH, Buco FH, Fiorelli LR, Issa V, Bacal F, Bocchi EA. Prophylactic donor tricuspid annuloplasty in orthotopic bicaval heart transplantation. Transplant Proc. 2007;39(8):2527-30.

12. Bacal F, Silva CP, Bocchi EA, et al. Mychophenolate mofetil increased Chagas' disease reactivation in heart transplanted patients: comparison between two different protocols. Am J Transplant. 2005;5:2017-21.

13. Bestetti RB, Souza TR, Lima MF, et al. Effects of a mycophenolate mofetil-based immunosuppressive regimen in Chagas' heart transplant recipients. Transplantation. 2007;84;3:441-2.

14. Corti M. AIDS and Chagas' disease. AIDS Patient Care STDS. 2000;14(11):581-8.

15. Gallerano V, Consigli J, Pereyra S, Gómez Zanni S, Danielo C, Gallerano RH, Guidi A. Chagas' disease reactivation with skin symptoms in a patient with kidney transplant. Int J Dermatol. 2007;46(6):607-10.

16. Fontes Rezende RE, Lescano MA, Zambelli Ramalho LN, de Castro Figueiredo JF, Oliveira Dantas R, Garzella Meneghelli U, Pimenta Módena JL. Reactivation of Chagas' disease in a patient with non-Hodgkin's lymphoma: gastric, oesophageal and laryngeal involvement. Trans $\mathrm{R}$ Soc Trop Med Hyg. 2006;100(1):74-8.

17. Altclas J, Sinagra A, Dictar M, Luna C, Verón MT, De Rissio AM, García MM, Salgueira C, Riarte A. Chagas disease in bone marrow transplantation: an approach to preemptive therapy. Bone Marrow Transplant. 2005;36(2):123-9.

18. Bacal F, Silva CP, Pires PV, Mangini S, Fiorelli AI, Stolf NG, Bocchi EA. Transplantation for Chagas' disease: an overview of immunosuppression and reactivation in the last two decades. Clin Transplant. 2010;24(2):E29-34.

19. Marchiori PE, Alexandre PL, Britto N, Patzina RA, Fiorelli AA, Lucato LT, Rosemberg S, Pereira SL, Stolf NG, Scaff M. Late reactivation of Chagas' disease presenting in a recipient as an expansive mass lesion in the brain after heart transplantation of chagasic myocardiopathy. J Heart Lung Transplant. 2007;26(11):1091-6.

20. Salvador F, Len O, Molina I, Sulleiro E, Sauleda S, Bilbao I, Castells L, Pont T, Gavaldà J, Pahissa A. Safety of liver transplantation performed with Chagas seropositive donors to seronegative recipients. Liver Transpl. 2011;17(11):1304-8.

21. Ortiz AM, Troncoso P, Sainz M, Vilches S. Prophylaxis and treatment of Chagas disease in renal transplant donor and recipient: case report. Transplant Proc. 2010;42(1):393-4.
22. Kun $\mathrm{H}$, Moore A, Mascola L, Steurer F, Lawrence G, Kubak B, Radhakrishna S, Leiby D, Herron R, Mone T, Hunter R, Kuehnert M; Chagas Disease in Transplant Recipients Investigation Team. Transmission of Trypanosoma cruzi by heart transplantation. Clin Infect Dis. 2009;48(11):1534-40.

23. Meneguetti JC, Camargo EE, Soares J Jr, Bellotti G, Bocchi EA, Higushi ML, Stolf NAG, Hironaka FH, Buchpiguel CA, Pileggi F. Gallium-67 imaging in human heart transplantation correlation with endomyocardial biopsy. J Heart Transplant. 1987;6(3):171-6.

24. Mehra MR, Crespo-Leiro MG, Dipchand A, Ensminger SM, Hiemann NE, Kobashigawa JA, Madsen J, Parameshwar J, Starling RC, Uber PA. International Society for Heart and Lung Transplantation working formulation of a standardized nomenclature for cardiac allograft vasculopathy-2010. J Heart Lung Transplant. 2010;29(7):717-27.

25. Benvenuti LAT, Roggério A, Sambiasea NV, Fiorelli Al, Higuchi ML. Polymerase chain reaction in endomyocardial biopsies for monitoring reactivation of Chagas' disease in heart transplantation. A case report and review of the literature. Cardiovasc Pathol. 2005; 14:265-8

26. Benvenuti LA, Roggério A, Coelho G, Fiorelli Al. Usefulness of qualitative polymerase chain reaction for Trypanosoma cruzi DNA in endomyocardial biopsy specimens of chagasic heart transplant patients. J Heart Lung Transplant. 2011;30(7):799-804.

27. Vila JHA, Macruz R, Sampaio FA, Pereira WI, Camargo M, Bittencourt D, Zerbini EJ. Transplante cardíaco em doença de Chagas: experiência preliminar. Arq Bras Cardiol. 1985;45(suppl I):1-132.

28. Almeida DR, Carvalho AC, Branco JN, Azevedo JE, Pires CA, Buffolo E, Martinez E. Transplante cardíaco em cardiomiopatia chagásica: experiência com esquema modificado de imunossupressão. Arq Bras Cardiol, 65(suppl I):1-19, 1995.

29. Bocchi EA, Fiorelli Al. The paradox of survival results after heart transplantation for cardiomyopathy caused by Trypanosoma cruzi. Ann Thorac Surg. 2001;71:1833-8.

30. Moreira LFP, Galantier J, Benício A, Leirner AA, Fiorelli Al, Stolf NAG, Oliveira SA. Perspectivas da evolução clínica de pacientes com cardiomiopatia chagásica listados em prioridade para o transplante cardíaco. Rev Bras Cir Cardiovasc. 2005;20(3):261-9.

31. Carvalho VB, Sousa EFL, Vila JHA, Silva JP, Caiado MR, Araujo SR, Macruz R, Zerbini EJ. Heart transplantation in Chagas' disease. 10 years after the initial experience. Circulation. 1996;94:1815-7. 